\title{
The characteristics of gastrointestinal symptoms in patients with severe COVID-19: a systematic review and meta-analysis
}

\author{
Yuki Hayashi $^{1} \cdot$ Kohei Wagatsuma $^{1} \cdot$ Masanori Nojima $^{2} \cdot$ Tsukasa Yamakawa $^{1} \cdot$ \\ Tadashi Ichimiya $^{1}$ - Yoshihiro Yokoyama ${ }^{1}$ - Tomoe Kazama ${ }^{1}$ Daisuke Hirayama ${ }^{1}$. \\ Hiroshi Nakase ${ }^{1}$ (D)
}

Received: 29 January 2021 / Accepted: 8 March 2021 / Published online: 23 March 2021

(C) Japanese Society of Gastroenterology 2021

\begin{abstract}
Although primarily a respiratory illness, several studies have shown that COVID-19 causes elevation of liver enzymes and various gastrointestinal (GI) symptoms. The aim of this study was to undertake a systematic review and meta-analysis to determine whether the presence of gastrointestinal (GI) symptoms contributed toward COVID-19 severity, and identify the GI symptoms characteristic of severe COVID-19. We conducted a literature search of PubMed from December 1, 2019, to June 30, 2020, and identified all reports with GI symptoms reported. A meta-analysis comparing the severity of COVID-19 with the presence of liver enzyme elevation and GI symptoms was performed using RevMan version 5.4. Pooled data from 15,305 unique reverse transcriptase-polymerase chain reaction positive COVID-19 patients from 44 studies were analyzed. We found that the severe COVID-19 patients significantly had abdominal pain compared to the non-severe COVID-19 patients $(\mathrm{OR}=2.70,95 \%$ CI $1.17-6.27, Z=2.32, p=0.02, \mathrm{I}^{2}=0 \%$ ) by analyzed 609 patients of 4 studies who reported both abdominal pain and COVID-19 severity. However, there was no significant difference in the incidence of diarrhea, nausea, or vomiting between the two groups. Thus, this systematic review and
\end{abstract}

Supplementary Information The online version contains supplementary material available at https://doi.org/10.1007/s00535021-01778-z.

Hiroshi Nakase

hiropynakase@gmail.com

1 Department of Gastroenterology and Hepatology, Sapporo Medical University of Medicine, S-1, W16, Chuoku, Sapporo, Hokkaido 060-8543, Japan

2 Center for Translational Research, The Institute of Medical Science, The University of Tokyo, Tokyo 108-8639, Japan meta-analysis demonstrated that abdominal pain could be characteristic of severe COVID-19 infections. Compared with other viral infections that primarily infect the respiratory system, patients with COVID-19 have a slightly lower frequency of diarrheal symptoms with abdominal pain. However, to confirm this, further studies with COVID-19 patients across various countries and ethnicities are required.

Keywords COVID-19 · Gastrointestinal system · Abdominal pain $\cdot$ Systematic review $\cdot$ Meta-analysis

\section{Introduction}

The outbreak of the novel coronavirus epidemic was first detected in Wuhan, China, in December 2019 and has since spread worldwide. The International Committee on Taxonomy of Viruses identified this novel virus as SARSCoV-2 [1], and the World Health Organization formally named this infectious disease as coronavirus disease 2019 (COVID-19) on February 2020 [2]. The severity of COVID-19 was mild in most patients (80-81\%), but $14 \%$ of patients experienced severe disease, and $5-6.1 \%$ of patients became critical [3, 4]. The most common symptoms of COVID-19 are fever, cough, and fatigue/myalgia [5]. Several reports have shown that patients with COVID19 have elevated liver enzymes and various gastrointestinal (GI) symptoms, such as diarrhea, abdominal pain, nausea, vomiting, and loss of appetite [6,7]. SARS-CoV-2 enters via cells expressing the angiotensin-converting enzyme 2 (ACE2) receptor. ACE2 is abundantly expressed in the glandular cells of the stomach, duodenum, small intestine, and colon, thereby supporting the theory that GI symptoms 
in patients with COVID-19 are triggered by the invasion of SARS-CoV-2 into host GI cells [8]. A recent systematic review of 78 studies with 12,797 patients found that the pooled prevalence of all GI symptoms was $17 \%$, the weighted pooled prevalence of diarrhea was $12.4 \%$, nausea and/or vomiting was $9.0 \%$, loss of appetite was $22.3 \%$, and abdominal pain was $6.2 \%$. Mortality among patients with GI symptoms was similar to the overall mortality. However, most studies had a high risk of bias, and the overall quality of evidence was either low or very low for all outcomes [9]. This study aimed to undertake a systematic review and meta-analysis of the existing literature on this subject to determine the association of COVID-19 severity with the presence of GI symptoms in COVID-19, and identify GI symptoms that are characteristic of severe COVID-19.

\section{Data and methods}

\section{Search strategy and selection criteria}

We conducted a literature search of PubMed from December 1, 2019, to June 30, 2020, to identify all studies with GI symptoms reported. The search formula was [(covid-19) OR (SARS-CoV-2) OR (novel coronavirus)] AND [(colon) OR (bowel) OR (hepato) OR (GI) OR (pancreas) OR (bladder) OR (liver) OR (gastrointestinal) OR (biliary) OR (diarrhea) OR (abdominal pain) OR (nausea) OR (vomiting) OR (anorexia) OR (jaundice)]. All search results were evaluated according to the guidelines of the Preferred Reporting Items for Systematic Reviews and Meta-Analyses statement [10].

The inclusion criteria were as follows: study population: patients diagnosed with COVID-19 (not include asymptomatic patients) by Reverse Transcription Polymerase Chain Reaction (RT-PCR) and classified according to the severity of COVID-19 or/and presence of elevated liver enzymes or/and presence of GI symptoms; study design: case series studies or cohort studies; outcomes measure: at least one of the following outcomes reported: GI symptoms, laboratory results, severity data, or past historical data. The exclusion criteria were as follows: abstract: conferences and commentary articles; population: data on pediatric patients or pregnant patients only.

Two independent authors ( $\mathrm{YH}$ and KW) screened the literature and assessed each study for inclusion. Any disagreements that arose were resolved through discussion.

\section{Data extraction and quality assessment}

Data extraction and literature quality assessment were conducted independently by one investigator ( $\mathrm{YH}$ ), which were rechecked for accuracy by a second reviewer (TI). Microsoft Excel 15.0 was used to record all available information, including age, sex, COVID-19 severity, GI symptoms (diarrhea, abdominal pain, nausea, vomiting, and anorexia), liver enzyme elevation [Total bilirubin (T.bil), aspartate transaminase (AST), alanine transaminase (ALT), alkaline phosphatase (ALP), and gamma-glutamyl transpeptidase $(\gamma-\mathrm{GTP})]$, and medical history (underlying diabetes mellitus (DM), liver disease, and GI disease). Studies with observed discrepancies in the data were excluded.

\section{Assessment of bias}

The Methodological Index for Non-Randomized Studies (MINORS) criteria was used to assess the risk of bias [11]. Each of the selected articles was scored as 0 (not reported), 1 (reported but inadequate), or 2 (reported and adequate). The highest possible score was 16 for non-comparative studies.

\section{Statistical analysis of data}

Microsoft Excel 15.0 was used to analyze the clinical symptoms and laboratory results. The meta-analysis was performed using Review Manager (RevMan) version 5.4.1 (The Cochrane Collaboration, 2020). Dichotomous data were analyzed using the Mantel-Haenszel method and the odds ratio (OR) and $95 \%$ confidence intervals $(95 \% \mathrm{CI})$ were obtained. Continuous data were analyzed using the inverse variance weighted method and expressed as mean differences with $95 \%$ CI. We used the $\mathrm{I}^{2}$ statistics to assess the magnitude of heterogeneity, and chose the appropriate effects model based on the results: the fixed effects model was used if $\mathrm{I}^{2}<50 \%$, and the random effects model was used if $\mathrm{I}^{2} \geq 50 \%$ [12-14]. For continuous data, the mean differences and standard deviations were incorporated into the calculations, but if the data were in quartiles, the medians were considered as the means, and the quartile deviations were converted to the standard deviation according to the Cochrane Handbook estimator $\left(\mathrm{SD} \approx \frac{q_{3}-q_{1}}{1.35}\right)[15]$

\section{Outcomes}

The primary objective of this meta-analysis was to identify GI symptoms characteristic of severe COVID-19. The secondary objective was to compare GI symptoms among patients with elevated liver enzymes and COVID-19, given that the relationship between elevated liver enzyme levels and critical COVID-19 has been previously reported [16]. Severe type of COVID-19 was defined as satisfying any of 


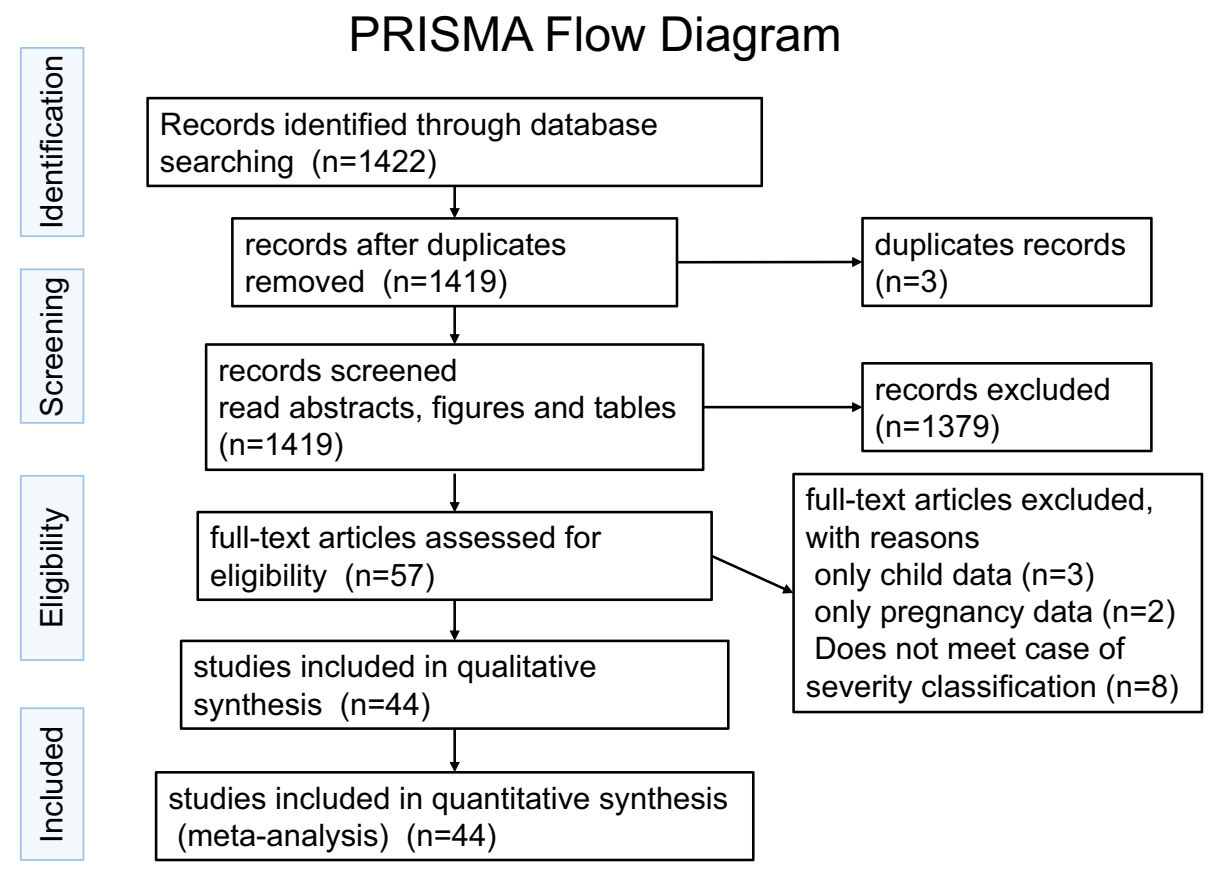

Fig. 1 PRISMA flow diagram [12]

the following items: (1) respiratory distress and respiratory frequency $>30 / \mathrm{min}$; (2) blood oxygen saturation $\leq 93 \%$ on room air; (3) $\mathrm{PaO}_{2} / \mathrm{FiO}_{2}$ ratio $\leq 300 \mathrm{mmHg}$; (4) lung infiltrates $>50 \%$ within $24-48 \mathrm{~h}$. Note that this definition is identical to the National Chinese Health Commission (seventh) edition definition [17] and the old World Health Organization (WHO) definition of severity [18] but differs from the latest WHO definition of severity $(\mathrm{SpO} 2<90 \%$, $\mathrm{RR}>30$, signs of severe respiratory distress (accessory muscle use, inability to complete full sentences, and, in children, very severe chest wall indrawing, grunting, central cyanosis, or presence of any other general danger signs) [19]. We included critical patients in "severe patients" in this review. Therefore, studies with inadequate separation of severe and non-severe COVID-19 in the cohort, such as comparing ICU patients and non-ICU patients, critical and non-critical COVID-19, were excluded in severity's meta-analysis. The upper limit of normal liver function varies slightly among countries and regions. The elevated liver function definition differs in each article. Therefore, this review does not attempt to define a uniform set of liver enzyme values. Since the quality of the literature included in the previous meta-analyses was low, we believed that a multifaceted review was required.

\section{Result}

\section{Study selection and quality assessment}

Our literature search produced 1422 publications. Of these, 1419 were unique records, of which 1369 were excluded after screening the abstracts, figures, and tables. The remaining 57 records were carefully and thoroughly evaluated. Thirteen studies were excluded for not having met the inclusion criteria. Finally, 44 records were included (Fig. 1) [20-66]. Based on our comprehensive selection process, we believe that the 44 included studies reported data on 15,305 unique COVID-19 patients. Most studies (90.9\%) in our analysis were from China, and the remaining four studies were from the USA (7.1\%), Italy (2.3\%), and France (2.3\%). The studies mainly included adults, although a few studies included a small proportion of pediatric patients. The study population comprised 12,250 patients with mild-moderate COVID-19, 3055 patients with severe COVID-19, and no asymptomatic carriers of SARS-CoV-2 (Supplement 2). The overall certainty in the body of evidence was low, since most of the studies were retrospective cohorts. The 44 eligible articles were subjected to a bias assessment using the MINORS criteria at the individual study level. The MINORS scores ranged from 6 to 12 (mean: 9.27, median: 9.0) (Supplement 3).

A meta-analysis was performed in two groups as follows: (1) COVID-19 severity (non-severe COVID-19 vs. 
Table 1 Summary of meta-analysis(1); outcome by the COVID-19 severity (severe COVID-19 vs. non-severe COVID-19)

\begin{tabular}{llll}
\hline Outcome & Studies & Participants & Effect estimate \\
\hline 1.1 GI symptoms (Not details described) & 4 & 692 & $1.10[0.68,1.78]$ \\
1.2 Diarrhea & 19 & 4322 & $1.26[0.99,1.61]$ \\
1.3 Abdominal pain & 4 & 609 & $2.70[1.17,6.27]$ \\
1.4 Nausea & 6 & 2199 & $1.04[0.50,2.16]$ \\
1.5 Vomiting & 5 & 1045 & $0.98[0.47,2.02]$ \\
1.6 Anorexia & 8 & 1097 & $1.70[0.90,3.20]$ \\
1.7T.Bil & 14 & 2482 & $0.14[0.07,0.21]$ \\
1.8 AST & 15 & 2571 & $11.41[7.20,15.62]$ \\
1.9 ALT & 13 & 2194 & $3.94[2.26,5.61]$ \\
1.10 $\gamma-$ GTP & 5 & 1168 & $6.99[1.32,12.66]$ \\
1.11 ALP & 4 & 846 & $-0.04[-4.16,4.09]$ \\
1.12 Liver enzyme elevation (described no details) & 4 & 544 & $4.01[2.47,6.51]$ \\
1.13 Past history liver & 17 & 9124 & $1.57[1.18,2.10]$ \\
1.14 Past history GI exclude liver & 2 & 170 & $2.76[1.10,6.89]$ \\
1.15 Past history DM & 22 & 3995 & $2.28[1.81,2.88]$ \\
\hline
\end{tabular}

severe COVID-19) (2) the presence of liver enzyme elevation.

\section{What are the symptoms characteristic of severe COVID-19?}

We assessed the GI and hepatic factors associated with a severe clinical course of infection (non-severe COVID-19 vs. severe COVID-19) (Table 1).

A total of four studies including 609 COVID-19 patients grouped by severity were included in the analysis for abdominal pain $[25,32,35,50]$. Patients with severe COVID-19 had a significantly lower incidence of abdominal pain than those non-severe illness (OR 2.70; [95\% CI 1.17-6.27; $\left.p=0.02 ; \mathrm{I}^{2}=0 \%\right]$ ) (Fig. 2).

In addition, the severity of COVID-19 in patients with elevated liver enzymes was significantly greater: T.bil (mean difference $0.14 \mathrm{mg} / \mathrm{dL} \quad[95 \%$ CI $0.07-0.21$; $\left.p<0.0001 ; \mathrm{I}^{2}=79 \%\right]$ ), AST (mean difference $11.41 \mathrm{IU} / \mathrm{L}$ [95\% CI 7.20-15.62; $\left.p<0.00001 ; \mathrm{I}^{2}=71 \%\right]$ ), ALT (mean difference 3.94 IU/L [95\% CI 2.26-5.61; $p<0.00001 ; \mathrm{I}^{2}=$ $18 \%]$ ), $\gamma$-GTP (mean difference, $6.99 \mathrm{IU} / \mathrm{L}$ [95\% CI $\left.\left.1.32-12.66 ; p=0.02 ; \mathrm{I}^{2}=0 \%\right]\right)$, and one elevated liver enzyme (no details described) (OR $4.01 \quad[95 \%$ CI 2.47-6.51; $\left.p<0.00001 ; \mathrm{I}^{2}=40 \%\right]$ ) (Supplementary figures A7-A10, A12).

Patients with a past history of liver disease, GI disease, or DM had significantly more severe COVID-19 compared to those without: liver disease (OR 1.57 [95\% CI $\left.1.18-2.10 ; p=0.002 ; \mathrm{I}^{2}=47 \%\right]$ ), GI disease (OR 2.76 [95\% CI 1.10-6.89; $\left.p=0.03 ; \mathrm{I}^{2}=0 \%\right]$ ), DM (OR 2.28 [95\% CI 1.81-2.88; $\left.p<0.00001 ; \mathrm{I}^{2}=0 \%\right]$ ) (Supplementary figures A13-A15). There was no significant difference in the presence of GI symptoms such as diarrhea, nausea, vomiting, anorexia, and elevation of ALP between patients with severe and non-severe COVID-19 (Supplementary figures A1, A2, A4, A5, A11).

\section{Analysis within the liver enzyme elevation group}

In the included reports, the most common definitions of liver enzyme elevation were T.bi $>1.0 \mathrm{mg} / \mathrm{dL}$, AST $>$ $30 \mathrm{IU} / \mathrm{L}, \quad$ ALT $>30 \mathrm{IU} / \mathrm{L}, \quad \gamma-\mathrm{GTP}>49 \mathrm{IU} / \mathrm{L}, \quad$ and ALP $>135$ IU/L. To determine factors associated with the presence of liver enzyme elevation, we assessed the contribution of GI symptoms and medical history (Table 2, Supplement-1 figures B1-B7). Lack of liver enzyme elevation was significantly associated with vomiting (OR 0.53 [95\% CI $0.31-0.92 ; p=0.02 ; \mathrm{I}^{2}=0 \%$ ), though not associated with diarrhea or nausea: diarrhea (OR 1.07; [95\% CI $\left.0.59-1.94 ; p=0.82 ; \mathrm{I}^{2}=50 \%\right]$ ) and nausea (OR $0.57[95 \%$ CI $\left.\left.0.32-1.01 ; p=0.05 ; \mathrm{I}^{2}=0 \%\right]\right)$. There were not enough studies to assess abdominal pain and anorexia. Liver enzyme elevation was associated with the past history of liver disease (OR 1.71; 95\% CI 1.13-2.58; $p=0.01 ; \mathrm{I}^{2}=$ $0 \%]$ ), but not with underlying DM (OR 0.87 [95\% CI $\left.\left.0.66-1.14 ; p=0.31 ; \mathrm{I}^{2}=0 \%\right]\right)$. The number of studies was insufficient to test the association of liver enzyme elevation with GI diseases.

\section{Analysis of the relationship between abdominal pain and diarrhea}

We examined the relationship between abdominal pain and diarrhea in all the screened articles with excluded articles in three meta-analyses since abdominal pain was 


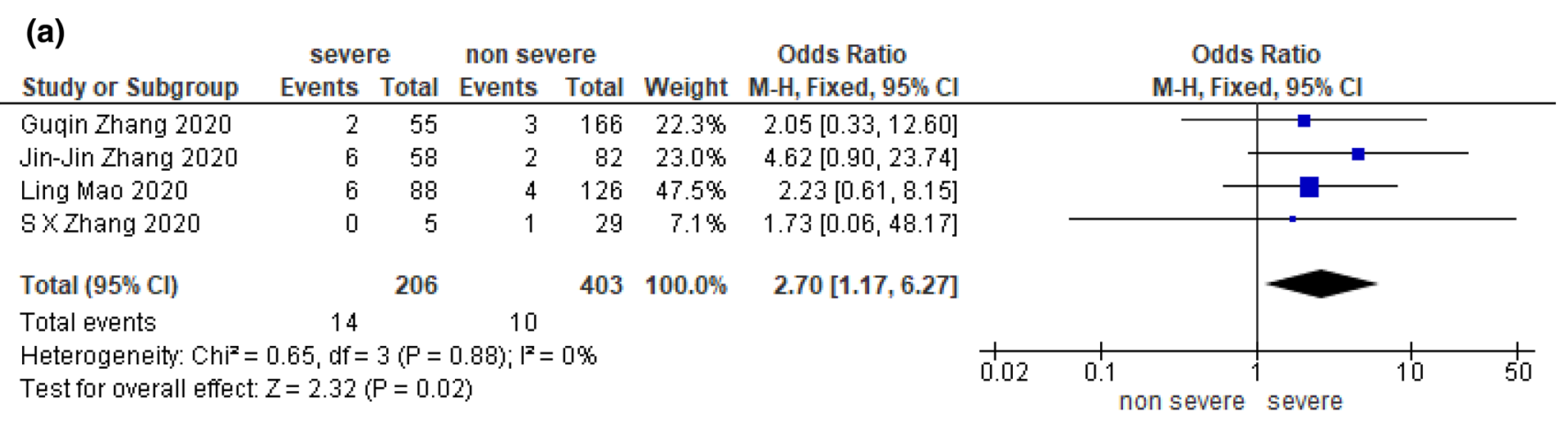

(b)

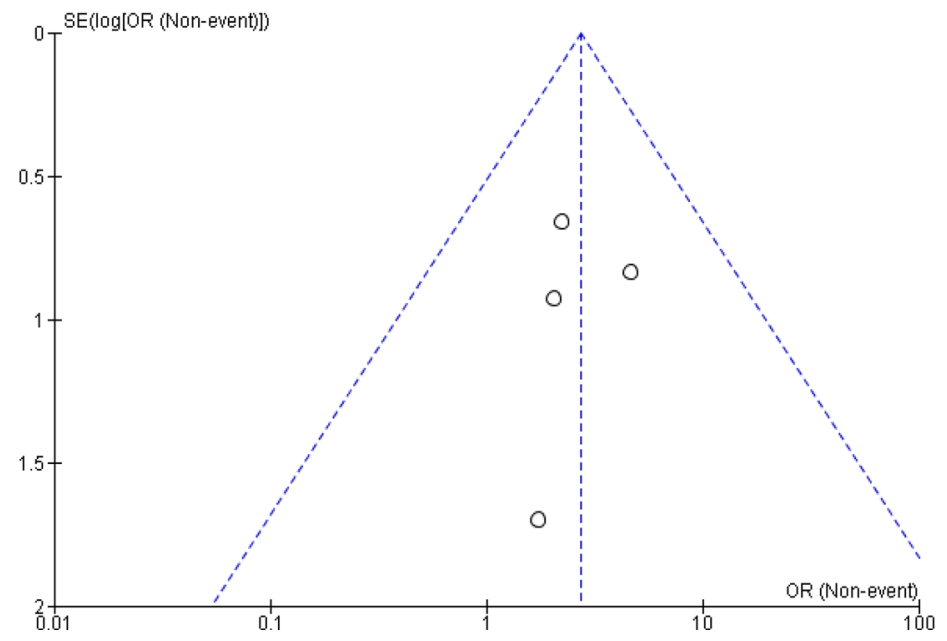

Fig. 2 Meta-analysis result; abdominal pain compared by COVID-19 severity. a Odds ratio, and forest plot. b Funnel plot [25, 32, 35, 50]

Table 2 Summary of meta-analysis (2); outcome by the presence of liver enzyme elevation (liver enzymes elevation vs. no elevation)

\begin{tabular}{lllr}
\hline Outcome & Studies & Participants & Effect estimate \\
\hline 2.1 Diarrhea & 7 & 1911 & $1.07[0.59,1.94]$ \\
2.2 Abdominal pain & 1 & 148 & $0.84[0.07,9.51]$ \\
2.3 Nausea & 3 & 1501 & $0.57[0.32,1.01]$ \\
2.4 Vomiting & 4 & 1681 & $0.53[0.31,0.91]$ \\
2.5 Anorexia & 1 & 156 & $0.35[0.04,3.20]$ \\
2.6 Past history liver & 5 & 2152 & $1.71[1.13,2.58]$ \\
2.7 Past history DM & 5 & 1053 & $0.87[0.66,1.14]$ \\
2.8 Past history GI exclude liver & 0 & 0 & Not estimable \\
\hline
\end{tabular}

characteristic of severe COVID-19. The ratio of abdominal pain to diarrhea was 0.23 (Table 3) [67-74]. This ratio was generally consistent with the regression line $(y=0.2442 x-$ $0.5447, R^{2}=0.554$ : least-squares method) (Fig. 3). In most of studies tabulated, the history of diarrhea and abdominal pain were collected independently. Therefore, the real extent of overlap between diarrhea and abdominal pain is unknown.

\section{Discussion}

Our meta-analysis demonstrated that the presence of abdominal pain was characteristic of severe COVID-19. This result was consistent with an existing meta-analysis on the relationship between abdominal pain and COVID-19 severity by Suresh et al. [75]. However, Suresh's systematic review [75] collected relatively early reports and did not sufficiently contrast mild-moderate COVID-19 and 
Table 3 The ratio abdominal pain to diarrhea in COVID-19 patients [68-75]

\begin{tabular}{llllll}
\hline First author & Country & Numeber of patients & Diarrhea $(n)$ & Abdominal pain $(n)$ & Abdominal pain/diarrhea ratio \\
\hline Jin-Jin Zhang & China & 140 & 18 & 8 & 0.44 \\
Q Guan & China & 8 & 3 & 1 & 0.33 \\
Pingzheng Mo & China & 155 & 7 & 3 & 0.43 \\
Ling Mao & China & 214 & 41 & 10 & 0.24 \\
Lei Pan & China & 204 & 35 & 2 & 0.06 \\
Zhenyu Fan & China & 148 & 6 & 3 & 0.50 \\
Chaoqun Han & China & 206 & 67 & 9 & 0.13 \\
Xiao-Shan Wei & China & 84 & 26 & 2 & 0.08 \\
Guqin Zhang & China & 221 & 25 & 5 & 0.20 \\
Walker D. Redd & USA & 318 & 107 & 46 & 0.43 \\
Xin-Ying Zhao & China & 91 & 14 & 2 & 0.14 \\
Elisabetta Buscarini & Italy & 411 & 15 & 5 & 0.33 \\
Xiao-Li Xiong & China & 244 & 15 & 4 & 0.27 \\
Ting Zheng & China & 1320 & 107 & 11 & 0.10 \\
Total & & 3764 & $486(13 \%)$ & $111(2.9 \%)$ & 0.23 \\
\hline
\end{tabular}

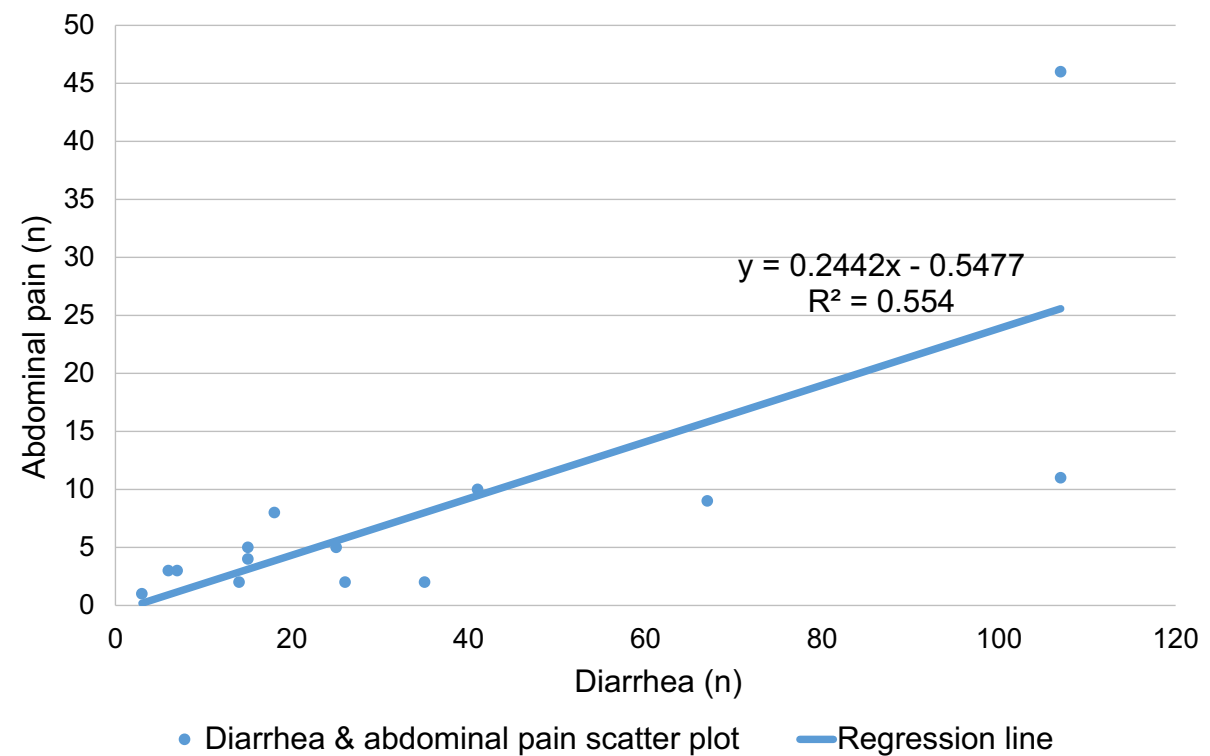

Fig. 3 The relationship between abdominal pain and diarrhea in COVID-19 patients (scatter plot and regression line) [68-75]. Based on Table 3 and this figure, we estimate that about $23 \%$ of diarrhea symptoms are accompanied by abdominal pain

severe critical COVID-19. In this meta-analysis, for analyzing abdominal pain and COVID-19 severity more purely, we excluded the two articles by Pan et al. and Wang et al. $[69,76]$ included in the meta-analysis by Suresh et al. [75]. Because, for assessing the severity of COVID-19, these reports compared admission ICU vs. non-ICU or discussed in a specific population. On the other hand, by increasing the number of included studies and patients, we could obtain a clear picture of the severity of abdominal pain and COVID-19 in symptomatic patients than existing reviews by extending the literature search period and checking all figures, including supplement material, during screening.

SARS-CoV-2, like SARS-CoV, has a spike protein on its surface. The enzyme protease cleaves the spike protein to bind to the ACE2 receptor on the surface of the infected cell, causing infection [77, 78]. It is known that transmembrane serine protease 2 (TMPRSS2), a type of serine 
protease expressed in membranes, is extremely efficient and can be activated in small amounts, and TMPRSS2 expression is higher in the intestinal tract and liver than in the lungs $[79,80]$. Moreover, in experiments with SARSCoV-2 using an artificial intestinal tract (enteroids), in addition to TMPRSS2, TMPRSS4 has been reported to proliferate during infection [81]. It is based on these laboratory findings that SARS-CoV-2 is hypothesized to cause severe GI symptoms. However, clinically, there have been few reports noting that the symptoms associated with SARS-CoV-2 intestinal infection are prognostic of COVID-19 outcomes.

Our meta-analysis demonstrated that among GI symptoms, abdominal pain was characteristic of severe COVID19 , while there was no significant difference in the incidence of diarrhea, nausea, vomiting, and anorexia between patients with mild-moderate and severe COVID-19. Besides, there was no significant difference in the severity of COVID-19 between patients with GI symptoms and those without. Moreover, our meta-analysis showed that patients with history of DM had a predominantly higher rate of severe disease. History of DM is generally a highrisk factor for thrombosis/vascular disease. Besides, Chen et al. reported that microthrombosis is one of the characteristic pathophysiologies of COVID-19, and $91.3 \%$ of dead patients had microthrombosis. They considered that endothelial damage caused by SARS-CoV-2 cell invasion with subsequent host immune response and activated coagulation pathways contributes the progression of severe COVID-19 [82]. These results may be related to the fact that a certain proportion of COVID-19 patients could experience intestinal upset and abdominal pain due to ischemic changes secondary to thrombosis caused by SARS-CoV-2 infection. SARS-CoV-2 causes thrombosis at various sites [83, 84]. In the NYC data, out of 12,630 hospitalized patients, 49 patients experienced thrombotic events, of which intestinal ischemia was reported in two cases [85]. Skok et al. reported that in autopsies of 19 COVID-19 patients, $30 \%$ of the patients had focal ischemic changes in the intestine [86]. Bhayana et al. [84] reported that the bowel findings included pneumatosis or portal venous gas, seen on $20 \%$ of CT images of patients in the ICU (4 of 20, the frequency of gastrointestinal abnormalities was significantly higher in ICU-admitted patients). In about half of them, intestinal ischemia was observed due to small vessel thrombosis. In other words, the intestinal damage caused by COVID-19 has a pattern of intestinal ischemia by thrombosis, in addition to the intestinal infection caused by SARS-CoV-2; thrombosis is more likely to occur in patients with severe COVID-19. Ischemic enteritis is reported to cause more abdominal pain than diarrhea or nausea/vomiting [87]. Our results suggest that the presence of abdominal pain in COVID-19 patients reflects intestinal ischemia caused by microthrombi and is an indicator of severe COVID-19. However, even in the presence of severe COVID-19-related bowel symptoms, there may be a reporting bias since significant respiratory failure, renal failure, and multiple organ failures are more notable.

A meta-analysis by Zheng et al. showed the high levels of D-dimer in patients with severe COVID-19 and the association between the levels and poor prognosis [88]. Therefore, if the abdominal pain is severe and the blood examination shows the abnormalities of coagulation test, contrast-enhanced CT should be performed. If there is thrombosis on $\mathrm{CT}$, thrombolytic therapy such as heparin should be performed. COVID-19 is often a microthrombus that may not be detected CT, therefore, if coagulation blood tests such as D-dimer are abnormal, antithrombotic therapy should be considered. The WHO recommends that DVT prophylaxis with heparin and other medications for all admission patients with COVID-19 [18]. However, Xiao et al. reported that ten of 60 patients with COVID-19 who had received antithrombotic treatment had GI bleeding [8]. Therefore, we should carefully consider antithrombotic therapy for COVID-19 patients with no apparent thrombus.

As for the elevation of liver enzymes in our metaanalysis, we found a statistically significant elevation of $\mathrm{T}$. bil, AST, ALT, and $\gamma$-GTP in patients with severe COVID19 than in those with mild-moderate COVID-19. The data in this current study were coincident with that of the previous meta-analysis [89]. On the other hand, there was no significant difference in the levels of T.bil, AST, and ALT between patients with GI symptoms and those without. These data may reflect that GI symptoms do not correlate with the severity of COVID-19 based on the elevation of liver enzymes. To investigate factors explaining liver enzyme elevation other than severe COVID-19, we performed a meta-regression analysis of age or history of DM and liver enzyme elevation in patients with COVID-19 severity (using EZR software with the package meta [90, 91]), but no significant results were obtained. These results may be due to the variable expression of ACE2 in various organs. Li et al. showed that the liver and lung have a similar distribution of ACE2 ribonucleic acid (RNA), and the colon has similar average ACE2 expression as the liver. Meanwhile, the small intestine has a high level of expression, approximately $10^{6}$ times higher than that in the liver, and the expression levels in the colon and small intestine vary compared to the liver and lung [92]. It is still unknown whether the organ-wise difference in ACE2 RNA expression is associated with corresponding symptoms of COVID-19. However, the similar levels of expression of the ACE2 RNA in the lung and liver may be the reason for 
the relevance of liver damage in the severity of COVID-19, that is mainly defined by respiratory symptoms.

We also found that the incidence of nausea and vomiting was significantly higher in COVID-19 patients without elevated liver enzymes than those with it. The patient groups with elevated liver enzymes mostly included patients with hepatitis B or non-alcoholic fatty liver disease (NAFLD). A meta-regression was performed for age and nausea or vomiting in the hepatic dysfunction group, but the results were not significantly different. Most body mass index (BMI) values were missing and could not be analyzed. However, BMI may be relevant, given that patients with liver enzyme elevation had a predominantly high BMI in the study by Hao et al. [47]. The mechanism by which COVID-19 causes nausea and vomiting is still unknown. In general, vomiting is caused by the stimulation of the vomiting center via the cerebral cortex, vestibular organs, chemoreceptor trigger zone, or the autonomic nervous system. Recently, the relationship between liver disease (hepatitis B, NAFLD, and others) and central nervous system (CNS) symptoms has been reported [93-95]. The significantly lower frequency of vomiting in the group with liver enzyme elevation might be associated with prior CNS changes due to liver disease, although the exact reasons remain unclear.

Next, we focused on the association between the severity of COVID-19 and comorbidities. There were significant differences in the severity of COVID-19 between patients with a history of liver disease, GI disease, and DM, and those without it, which is consistent with the results of the existing meta-analyses [88, 96]. Uchida et al. [97] reported that fatty liver, which can be identified by $\mathrm{CT}$, is associated with severe COVID-19 and elevated liver function enzymes. On the other hand, a history of GI disease or DM did not contribute to elevated liver enzymes and GI symptoms in patients with COVID-19. Targher et.al. reported that DM was not a risk factor in the association between metabolic dysfunction associated fatty liver disease and severe COVID-19 [98]. Portincasa et al. discussed the possible involvement of COVID-19 in inflammatory pathways in metabolically compromised NAFLD patients who could have a poor COVID-19 outcomes, including acute liver damage [99]. Thus, the association between a history of DM and elevated liver function enzymes in COVID-19 remains controversial.

We investigated the relationship between diarrhea and abdominal pain in COVID-19 patients. In this study, there were no studies that defined diarrhea clearly, except for the report by Wei et al. [71] We found that 2.9\% of COVID-19 patients had abdominal pain, $13 \%$ had diarrhea, and the ratio of abdominal pain to diarrhea was approximately 0.25 . Our data collection had the incidence of abdominal pain and diarrhea but had no relationship between two symptoms. Therefore, we adopt "the ratio of abdominal pain to diarrhea" to discuss the relationship between diarrhea and abdominal pain. However, we thought this was insufficient to show the relationship between the two, so we added the regression curve as a compliment. The ratios of abdominal pain and diarrhea are very close to each calculation, which is $0.23-0.24$. Based on these findings, we estimate that about $23 \%$ of diarrhea symptoms are accompanied by abdominal pain. Zayet et al. [100] reported that COVID-19 patients had a significantly higher incidence of diarrhea compared to influenza $\mathrm{A} / \mathrm{B}$ patients. However, the frequency of abdominal pain was not significantly different between patients with COVID-19 and influenza $\mathrm{A} / \mathrm{B}$. The ratio of abdominal pain to diarrhea in COVID-19 patients was 0.5, while the ratio in influenza $\mathrm{A} / \mathrm{B}$ patients was 0.82 . Although the incidence of abdominal pain and diarrhea in COVID-19 patients varied widely in the published literature, abdominal pain with diarrhea is likely to be a less common feature of COVID-19.

In patients with severe acute respiratory syndrome (SARS, which infects via ACE2 receptors), 3.9\% of patients had abdominal pain and $7.5 \%$ had diarrhea, and the ratio of abdominal pain to diarrhea was 0.52 [101]. In Middle East respiratory syndrome (which infects via DDP4 [102]), $17 \%$ of patients had abdominal pain and $25.5 \%$ had diarrhea, and the ratio of abdominal pain to diarrhea was 0.58 [103]. In norovirus infections, $59 \%$ of patients had abdominal pain and $68 \%$ had diarrhea, and the ratio of abdominal pain to diarrhea was 0.862 [104, 105]. In summary, COVID-19 has a lower ratio of abdominal pain to diarrhea than these infections, that is, lesser incidence of abdominal pain even in the presence of diarrheal symptoms. Generally, viral infections cause an alteration in intestinal permeability, resulting in enterocyte dysfunction and diarrhea. However, the mechanism of abdominal pain is complex and includes abnormalities in intestinal peristalsis, intestinal edema, and intestinal ischemia. The cause of the discrepancy between abdominal pain and diarrheal symptoms in COVID-19 is not well understood. However, a similar phenomenon of divergence between abdominal pain and diarrheal symptoms was reported in common acute respiratory infections (ARI). Minodier et al. [106] reported that diarrhea was observed in $14.6 \%$ of 574 patients with ARI and abdominal pain in $34.3 \%$, and the ratio of abdominal pain to diarrhea was 2.35 . They performed RT-PCR on nasal swabs and stool to look for pathogenic organisms. Despite accounting for the fact that some patients with multiple viral infections such as enteroviruses and children under 15 years of age (18\% of all patients) were included, the frequency of abdominal pain was higher than that of diarrhea. In addition, 28 patients were detected with coronavirus (NL63, 229E, OC43, and HKU1 strains, which are common human coronaviruses), 
and $78.6 \%$ of them had GI symptoms. If these results were valid worldwide, then commoner viral infections could cause GI symptoms more frequently than expected. There seems to be a divergence between diarrhea and abdominal pain.

Interestingly, Singh et al. [107] reported a difference in the incidence of diarrhea and abdominal pain in a cohort of COVID-19 patients based on the history of inflammatory bowel disease (IBD). They reported that the ratio of abdominal pain to diarrhea in IBD patients with COVID-19 was higher than that in non-IBD patients ( 0.95 vs. 0.53 , with no significant difference between the two groups on the $t$ test.). The reason for this phenomenon remains unclear. However, the epithelium in IBD patients who are in clinical remission is already 'primed' by a low level of inflammation related to IBD. Thus, in these patients, viral infection can easily trigger a further increase in intestinal permeability by fostering further recruitment of lymphocytes into the intestinal mucosa and by production of proinflammatory cytokines. Based on these findings, it would be reasonable to assume that IBD patients with COVID-19 have more abdominal symptoms than those without IBD.

An NYC cohort data showed that ethnic minority groups were at risk for COVID-19 deaths and intensive care unit (ICU) admissions [108]. However, a systematic review could not confirm that ethnicity was an independent poor prognostic factor for COVID-19 after adjusting for comorbidities [109]. There is no detailed report on the difference between ethnic/race and GI symptoms at present. In this study, we were able to examine the incidence of diarrheal symptoms of COVID-19 in China, the United States, and Italy (Table.3), but there was no tendency for the incidence of diarrhea to differ among the nation.

This study has several limitations. We researched articles containing abstracts written in English. Therefore, we might miss out on some reports written in non-English. Many studies of COVID-19 lack definitions of GI symptoms. However, the similar tendency is observed in reports regarding respiratory infections such as SARS, MERS, and flu. We consider that this is a common limitation of the study, which exam GI symptoms in respiratory infections. In most studies tabulated, diarrhea and abdominal pain were collected independently, and the true rate of overlap between diarrhea and abdominal pain is unknown. Most of the patients in this meta-analysis were from China and most likely of Asian descent. The number of studies to be included was 3 or 4 , and the result might change if the number of studies covered increases. The results may be prone to bias due to the exclusion of pediatric and pregnant patients. It has been noted that in children, the frequency of diarrhea and vomiting differs by age groups, even for the same enteroviral infection [110]; it is thus necessary to evaluate symptoms by age. In addition, the proportion of severe COVID-19 patients was higher than that in common SARS-CoV-2 infections, because our study included many case series. There were many missing values, and confounding factors could not be analyzed.

In conclusion, our study has shown that abdominal pain is likely to be characteristic of severe COVID-19. Compared with other viral infectious diseases that primarily infect the respiratory system, patients with COVID-19 may have a slightly lower frequency of diarrheal symptoms with abdominal pain. Since most of the patients in this study were from China, future studies should include patients from various countries and ethnicities.

Author contributions YY and TK conceived the study and designed the protocol with YH, TY, and TI. YH and KW conducted study selection and data extraction. $\mathrm{YH}$ and $\mathrm{MN}$ contributed to statistical analysis and interpretation of data. $\mathrm{YH}, \mathrm{DH}$, and $\mathrm{HN}$ drafted the manuscript with all authors critically revising the manuscript.

Funding This work was supported in part by Health and Labour Sciences Research Grants for research on intractable diseases from the Ministry of Health, Labour and Welfare of Japan (Investigation and Research for intractable Inflammatory Bowel Disease to HN), and Japan Society for the Promotion of Science Grants-in-Aid for Scientific Research grants: JSPS KAKENHI Grant Number JP 18 H02799 (to HN). The funders of the study had no role in the study design, data collection, data analysis, data interpretation, or writing of the report.

\section{Declarations}

Conflict of interest All authors declare that there is no conflict of interest.

\section{References}

1. Gorbalenya AE, Baker SC, Baric RS, et al. The species Severe acute respiratory syndrome-related coronavirus: classifying 2019-nCoV and naming it SARS-CoV-2. Nat Microbiol Nat Publ Group. 2020;5:536-44.

2. World Health Organization. Novel Coronavirus (2019-nCoV) Situation Report - 22 [Internet]. Nov. Coronavirus2019-NCoV Situat. Rep. - 22. 2020 [cited 2020 Sep 26]. Available from; https://www.who.int/docs/default-source/coronaviruse/situationreports/20200211-sitrep-22-ncov.pdf

3. Wu Z, McGoogan JM. Characteristics of and important lessons from the coronavirus disease 2019 (COVID-19) outbreak in China: summary of a report of 72314 cases from the Chinese center for disease control and prevention. JAMA Am Med Assoc. 2020;323:1239-42.

4. Organization WH. Coronavirus disease 2019 (COVID-19): situation report, 82. World Health Organization; 2020 [cited 2020 Dec 7]; Available from; https://apps.who.int/iris/handle/10665/ 331780

5. Ghayda RA, Lee J, Lee JY, et al. Correlations of clinical and laboratory characteristics of COVID-19: a systematic review and meta-analysis. Int J Environ Res Publ Health. 2020;17:1.

6. Parasa S, Desai M, Thoguluva Chandrasekar V, et al. Prevalence of gastrointestinal symptoms and fecal viral shedding in patients 
with coronavirus disease 2019. JAMA Netw Open. 2020;3:e2011335.

7. Sultan S, Altayar O, Siddique SM, et al. AGA institute rapid review of the gastrointestinal and liver manifestations of COVID-19, meta-analysis of international data, and recommendations for the consultative management of patients with COVID-19. Gastroenterology. 2020;159(320-334):e27.

8. Xiao F, Tang M, Zheng X, et al. Evidence for gastrointestinal infection of SARS-CoV-2. Gastroenterology. 2020;158(1831-1833):e3.

9. Tariq R, Saha S, Furqan F, et al. Prevalence and mortality of COVID-19 patients with gastrointestinal symptoms: a systematic review and meta-analysis. Mayo Clin Proc. 2020;95:1632-48.

10. Moher D, Liberati A, Tetzlaff J, et al. Preferred reporting items for systematic reviews and meta-analyses: the PRISMA statement. PLOS Med Publ Libr Sci. 2009;6:e1000097.

11. Slim K, Nini E, Forestier D, et al. Methodological index for nonrandomized studies (MINORS): development and validation of a new instrument. ANZ J Surg. 2003;73:712-6.

12. Greenland S. Quantitative methods in the review of epidemiologic literature. Epidemiol Rev Oxford Acad. 1987;9:1-30.

13. DerSimonian R, Laird N. Meta-analysis in clinical trials. Control Clin Trials. 1986;7:177-88.

14. Zhang Z, Wu P, Zhang J, et al. The effect of statins on microalbuminuria, proteinuria, progression of kidney function, and all-cause mortality in patients with non-end stage chronic kidney disease: a meta-analysis. Pharmacol Res. 2016;105:74-83.

15. Chapter 6: Choosing effect measures and computing estimates of effect [Internet]. [cited 2020 Sep 24]. Available from; / handbook/current/chapter-06

16. Xu L, Mao Y, Chen G. Risk factors for 2019 novel coronavirus disease (COVID-19) patients progressing to critical illness: a systematic review and meta-analysis. Aging. 2020;12:12410-21.

17. Chinese Clinical Guidance for COVID-19 Pneumonia Diagnosis and Treatment (7th edition) [Internet]. [cited 2020 Oct 1]. Available from; http://kjfy.meetingchina.org/msite/news/show/ $\mathrm{cn} / 3337 . \mathrm{html}$

18. World Health Organization. Clinical management of severe acute respiratory infection (SARI) when COVID-19 disease is suspected. Interim guidance. Pediatr Med Rodz. 2020;16:9-26.

19. Therapeutics and COVID-19: living guideline [Internet]. [cited 2021 Feb 16]. Available from; https://www.who.int/publica tions-detail-redirect/therapeutics-and-covid-19-living-guideline

20. Lee BT, Perumalswami PV, Im GY, et al. COVID-19 in liver transplant recipients: an initial experience from the US epicenter. Gastroenterology. 2020;159(1176-1178):e2.

21. Zhou C, Huang Z, Tan W, et al. Predictive factors of severe coronavirus disease 2019 in previously healthy young adults: a single-center, retrospective study. Respir Res. 2020;21:157.

22. Cao C, Chen M, He L, et al. Clinical features and outcomes of COVID-19 patients with gastrointestinal symptoms. Crit Care. 2020;24:340.

23. Chuan L, Zicheng J, Chuxiao S, et al. Preliminary study of the relationship between novel coronavirus pneumonia and liver function damage: a multicenter study. Chin J Hepatol. 2020;28:148-52 ((Chinese Medical Journals Publishing House Co., Ltd.)).

24. Lei F, Liu Y-M, Zhou F, et al. Longitudinal association between markers of liver injury and mortality in COVID-19 in China. Hepatology. 2020;72:389-98.

25. Zhang G, Hu C, Luo L, et al. Clinical features and short-term outcomes of 221 patients with COVID-19 in Wuhan. China $\mathrm{J}$ Clin Virol. 2020;127:104364.
26. Xie H, Zhao J, Lian N, et al. Clinical characteristics of non-ICU hospitalized patients with coronavirus disease 2019 and liver injury: a retrospective study. Liver Int. 2020;40:1321-6.

27. Shi H, Wang W, Yin J, et al. The inhibition of IL-2/IL-2R gives rise to CD8 + T cell and lymphocyte decrease through JAK1STAT5 in critical patients with COVID-19 pneumonia. Cell Death Dis Nat Publ Group. 2020;11:1-8.

28. Zhang H, Wang L, Chen Y, et al. (2020) Outcomes of novel coronavirus disease 2019 (COVID-19) infection in 107 patients with cancer from Wuhan, China. Cancer [Internet]. 2020 [cited 2020 Sep 28]; Available from; https://www.ncbi.nlm.nih.gov/ pmc/articles/PMC7361610/

29. Zhang H, Liao Y-S, Gong J, et al. Clinical characteristics of coronavirus disease (COVID-19) patients with gastrointestinal symptoms: a report of 164 cases. Dig Liver Dis. 2020;52:1076-9.

30. Gong J, Ou J, Qiu X, et al. A tool for early prediction of severe coronavirus disease 2019 (COVID-19): a multicenter study using the risk Nomogram in Wuhan and Guangdong, China. Clin Infect Dis Oxford Acad. 2020;71:833-40.

31. Huang J-T, Ran R-X, Lv Z-H, et al. Chronological changes of viral shedding in adult inpatients with COVID-19 in Wuhan, China. Clin Infect Dis. 2020;71:2158-66.

32. Zhang J, Dong X, Cao Y, et al. Clinical characteristics of 140 patients infected with SARS-CoV-2 in Wuhan, China. Allergy. 2020;75:1730-41.

33. Li J, Chen Z, Nie Y, et al. Identification of symptoms prognostic of COVID-19 severity: multivariate data analysis of a case series in Henan Province. J Med Internet Res. 2020;22:e19636.

34. Sun L, Shen L, Fan J, et al. Clinical features of patients with coronavirus disease 2019 from a designated hospital in Beijing, China. J Med Virol. 2020;92:2055-66.

35. Mao L, Jin $\mathrm{H}$, Wang $\mathrm{M}$, et al. Neurologic manifestations of hospitalized patients with coronavirus disease 2019 in Wuhan, China. JAMA Neurol Am Med Assoc. 2020;77:683-90.

36. Lin L, Jiang X, Zhang Z, et al. Gastrointestinal symptoms of 95 cases with SARS-CoV-2 infection. Gut BMJ Publ Group. 2020;69:997-1001.

37. Meszaros M, Meunier L, Morquin D, et al. Abnormal liver tests in patients hospitalized with coronavirus disease 2019: should we worry? Liver Int. 2020;40:1860-4.

38. Pereira MR, Mohan S, Cohen DJ, et al. COVID-19 in solid organ transplant recipients: initial report from the US epicenter. Am J Transpl. 2020;20:1800-8.

39. Min L, Peng H, Huiguo L, et al. Clinical characteristics of 30 medical workers infected with new coronavirus pneumonia. Chin J Tuber Respir Dis. 2020;43:E016-E016 ((Chinese Medical Journals Publishing House Co., Ltd.)).

40. Na Y, Suna W, Jianqi L, et al. Clinical characteristics and influencing factors of patients with novel coronavirus pneumonia combined with liver injury in Shaanxi region. Chin J Hepatol. 2020;28:E003-E003 ((Chinese Medical Journals Publishing House Co., Ltd.)).

41. Li Q, Zhang J, Ling Y, et al. A simple algorithm helps early identification of SARS-CoV-2 infection patients with severe progression tendency. Infection. 2020;48:577-84.

42. Chen Q, Zheng Z, Zhang C, et al. Clinical characteristics of 145 patients with corona virus disease 2019 (COVID-19) in Taizhou, Zhejiang, China. Infection. 2020;48:543-51.

43. Cai Q, Huang D, Yu H, et al. COVID-19: abnormal liver function tests. J Hepatol. 2020;73:566-74.

44. Yang Q, Xie L, Zhang W, et al. Analysis of the clinical characteristics, drug treatments and prognoses of 136 patients with coronavirus disease 2019. J Clin Pharm Ther. 2020;45:609-16. 
45. Wang Q, Zhao H, Liu L-G, et al. Pattern of liver injury in adult patients with COVID-19: a retrospective analysis of 105 patients. Mil Med Res. 2020;7:28.

46. Piano S, Dalbeni A, Vettore E, et al. Abnormal liver function tests predict transfer to intensive care unit and death in COVID19. Liver Int. 2020;40:2394-406.

47. Hao S-R, Zhang S-Y, Lian J-S, et al. Liver enzyme elevation in coronavirus disease 2019: a multicenter, retrospective, crosssectional study. Off $\mathrm{J}$ Am Coll Gastroenterol ACG. 2020;115:1075-83.

48. Sun D-Q, Wang T-Y, Zheng KI, et al. Subclinical acute kidney injury in COVID-19 patients: a retrospective cohort study. Nephron Karger Publ. 2020;144:347-50.

49. Wan S, Xiang Y, Fang W, et al. Clinical features and treatment of COVID-19 patients in northeast Chongqing. J Med Virol. 2020;92:797-806.

50. Shuxiang Z, Jing L, Wei Z, et al. The analysis of clinical characteristics of 34 novel coronavirus pneumonia cases in Ningxia Hui autonomous region. Chin J Tuberc Respir Dis. 2020;43:E037-E037 ((Chinese Medical Journals Publishing House Co., Ltd.)).

51. Liu T, Zhang J, Yang Y, et al. The role of interleukin-6 in monitoring severe case of coronavirus disease 2019. EMBO Mol Med. 2020;12:e12421 ((John Wiley \& Sons, Ltd)).

52. Redd WD, Zhou JC, Hathorn KE, et al. Prevalence and characteristics of gastrointestinal symptoms in patients with severe acute respiratory syndrome coronavirus 2 infection in the United States: a multicenter cohort study. Gastroenterology. 2020;159(765-767):e2.

53. Guan W, Ni Z, Hu Y, et al. Clinical characteristics of coronavirus disease in China. $N$ Engl $J$ Med. 2019;2020382:1708-20.

54. Zou X, Fang M, Li S, et al. Characteristics of liver function in patients with SARS-CoV-2 and chronic HBV coinfection. Clin Gastroenterol Hepatol. 2021;19:597-603.

55. Qi X, Liu C, Jiang Z, et al. Multicenter analysis of clinical characteristics and outcomes in patients with COVID-19 who develop liver injury. J Hepatol. 2020;73:455-8 ((Elsevier)).

56. Jin X, Lian J-S, Hu J-H, et al. Epidemiological, clinical and virological characteristics of 74 cases of coronavirus-infected disease 2019 (COVID-19) with gastrointestinal symptoms. Gut BMJ Publ Group. 2020;69:1002-9.

57. Zhao X-Y, Xu X-X, Yin H-S, et al. Clinical characteristics of patients with 2019 coronavirus disease in a non-Wuhan area of Hubei Province, China: a retrospective study. BMC Infect Dis. 2020;20:311.

58. Zhang Y, Zheng L, Liu L, et al. Liver impairment in COVID-19 patients: a retrospective analysis of 115 cases from a single centre in Wuhan city, China. Liver Int. 2020;40:2095-103.

59. Yudong $\mathrm{P}$, Kai M, Hongquan G, et al. Clinical characteristics and outcomes of 112 cardiovascular disease patients infected by 2019-nCoV. Chin J Cardiol. 2020;48:E004-E004 ((Chinese Medical Journals Publishing House Co., Ltd.)).

60. Wang Y, Liu S, Liu H, et al. SARS-CoV-2 infection of the liver directly contributes to hepatic impairment in patients with COVID-19. J Hepatol. 2020;73:807-16 ((Elsevier)).

61. Sun Y, Dong Y, Wang L, et al. Characteristics and prognostic factors of disease severity in patients with COVID-19: the Beijing experience. J Autoimmun. 2020;112:102473.

62. Zhou Y, He Y, Yang H, et al. Development and validation a nomogram for predicting the risk of severe COVID-19: a multicenter study in Sichuan, China. PLoS ONE. 2020;15:e0233328.

63. Zhou Y-J, Zheng KI, Wang X-B, et al. Metabolic-associated fatty liver disease is associated with severity of COVID-19. Liver Int. 2020;40:2160-3.
64. Cao Z, Li T, Liang L, et al. Clinical characteristics of Coronavirus Disease 2019 patients in Beijing, China. PLoS ONE. 2020;15:e234764 ((Public Library of Science)).

65. Fan Z, Chen L, Li J, et al. Clinical features of COVID-19-related liver functional abnormality. Clin Gastroenterol Hepatol. 2020;18:1561-6.

66. Zhiping Q, Xue M, Yuyi Z, et al. Analysis of baseline liver biochemical parameters in 324 cases with novel coronavirus pneumonia in Shanghai area. Chin J Hepatol. 2020;28:E005E005 ((Chinese Medical Journals Publishing House Co., Ltd.)).

67. Qun G, Miao L, Yingjie Z, et al. Epidemiological investigation of a family clustering of COVID-19. Chin J Epidemiol. 2020;41:629-33 ((Chinese Medical Journals Publishing House Co., Ltd.)).

68. Mo P, Xing Y, Xiao Y, et al. (2020) Clinical characteristics of refractory COVID-19 pneumonia in Wuhan, China. Clin Infect Dis [Internet]; Available from; https://academic.oup.com/cid/ advance-article/. doi: https://doi.org/10.1093/cid/ciaa270/ 5805508

69. Pan L, Mu M, Yang P, et al. Clinical characteristics of COVID19 patients with digestive symptoms in Hubei, China: a descriptive, cross-sectional, multicenter study. Off J Am Coll Gastroenterol ACG. 2020;115:766-73.

70. Han C, Duan C, Zhang S, et al. Digestive symptoms in COVID19 patients with mild disease severity: clinical presentation, stool viral RNA testing, and outcomes. Off J Am Coll Gastroenterol ACG. 2020;115:916-23.

71. Wei X-S, Wang X, Niu Y-R, et al. Diarrhea is associated with prolonged symptoms and viral carriage in corona virus disease 2019. Clin Gastroenterol Hepatol. 2020;18(1753-1759):e2.

72. Buscarini E, Manfredi G, Brambilla G, et al. GI symptoms as early signs of COVID-19 in hospitalised Italian patients. Gut BMJ Publ Group. 2020;69:1547-8.

73. Xiong X, Wong KK, Chi S, et al. (2020) Comparative study of the clinical characteristics and epidemiological trend of 244 COVID-19 infected children with or without GI symptoms. Gut [Internet]. BMJ Publishing Group; 2020 [cited 2020 Oct 1]; Available from; https://gut.bmj.com/content/early/2020/06/02/ gutjnl-2020-321486

74. Zheng T, Yang C, Wang H-Y, et al. Clinical characteristics and outcomes of COVID-19 patients with gastrointestinal symptoms admitted to Jianghan Fangcang Shelter Hospital in Wuhan, China. J Med Virol. 2020;92:2735-41.

75. Suresh Kumar VC, Mukherjee S, Harne PS, et al. Novelty in the gut: a systematic review and meta-analysis of the gastrointestinal manifestations of COVID-19. BMJ Open Gastroenterol. 2020;7:e000417.

76. Wang D, Hu B, Hu C, et al. Clinical characteristics of 138 hospitalized patients with 2019 novel coronavirus-infected pneumonia in Wuhan, China. JAMA. 2020;323:1061-9.

77. Belouzard S, Chu VC, Whittaker GR. Activation of the SARS coronavirus spike protein via sequential proteolytic cleavage at two distinct sites. Proc Natl Acad Sci. 2009;106:5871-6 ((National Academy of Sciences)).

78. Lan J, Ge J, Yu J, et al. Structure of the SARS-CoV-2 spike receptor-binding domain bound to the ACE2 receptor. Nature. 2020;581:215-20.

79. Millet JK, Whittaker GR. Host cell entry of Middle East respiratory syndrome coronavirus after two-step, furin-mediated activation of the spike protein. Proc Natl Acad Sci. 2014;111:15214-9 ((National Academy of Sciences)).

80. Ou X, Liu Y, Lei X, et al. Characterization of spike glycoprotein of SARS-CoV-2 on virus entry and its immune cross-reactivity with SARS-CoV. Nat Commun. 2020;11:1620 ((Nature Publishing Group)). 
81. Zang R, Castro MFG, McCune BT, et al. TMPRSS2 and TMPRSS4 promote SARS-CoV-2 infection of human small intestinal enterocytes. Sci Immunol. 2020;5:eabc3582 ((Science Immunology)).

82. Chen W, Pan JY. Anatomical and pathological observation and analysis of SARS and COVID-19: microthrombosis is the main cause of death. Biol Proced Online. 2021;23:4.

83. Gartland RM, Velmahos GC. Bowel necrosis in the setting of COVID-19. J Gastrointest Surg. 2020;1:1-2.

84. Bhayana R, Som A, Li MD, et al. Abdominal imaging findings in COVID-19: preliminary observations. Radiol Radiol Soc N Am. 2020;297:E207-15.

85. Etkin Y, Conway AM, Silpe J, et al. Acute arterial thromboembolism in patients with COVID-19 in the New York City area. Ann Vasc Surg. 2021;70:290-4.

86. Skok K, Stelzl E, Trauner M, et al. Post-mortem viral dynamics and tropism in COVID-19 patients in correlation with organ damage. Virchows Arch Int J Pathol. 2020;1:1-11.

87. Okawa K, Kitano A, Nakamura S, et al. Clinical study of ischemic colitis. Gastroenterol Endosc. 1990;32:365-376_1.

88. Zheng Z, Peng F, Xu B, et al. Risk factors of critical and mortal COVID-19 cases: a systematic literature review and metaanalysis. J Infect. 2020;81:e16-25.

89. Kumar-M P, Mishra S, Jha DK, et al. Coronavirus disease (COVID-19) and the liver: a comprehensive systematic review and meta-analysis. Hepatol Int. 2020;14:711-22.

90. Kanda Y. Investigation of the freely available easy-to-use software 'EZR' for medical statistics. Bone Marrow Transpl. 2013;48:452-8 ((Nature Publishing Group)).

91. Schwarzer G, Carpenter J, Rücker G (2015) Meta-analysis with R [Internet]. Springer international publishing; 2015. Available from; https://www.springer.com/de/book/9783319214153

92. Li M-Y, Li L, Zhang Y, et al. Expression of the SARS-CoV-2 cell receptor gene ACE2 in a wide variety of human tissues. Infect Dis Poverty. 2020;9:45.

93. Mastroeni D, Nolz J, Sekar S, et al. Laser-captured microglia in the Alzheimer's and Parkinson's brain reveal unique regional expression profiles and suggest a potential role for hepatitis B in the Alzheimer's brain. Neurobiol Aging. 2018;63:12-21.

94. Takahashi A, Kono S, Wada A, et al. Reduced brain activity in female patients with non-alcoholic fatty liver disease as measured by near-infrared spectroscopy. PLoS ONE. 2017;12:e0174169.

95. Kim D-G, Krenz A, Toussaint LE, et al. Non-alcoholic fatty liver disease induces signs of Alzheimer's disease (AD) in wildtype mice and accelerates pathological signs of $\mathrm{AD}$ in an $\mathrm{AD}$ model. J Neuroinflammation. 2016;13:1.

96. Wang B, Li R, Lu Z, et al. Does comorbidity increase the risk of patients with COVID-19: evidence from meta-analysis. Aging. 2020;12:6049-57.

97. Uchida Y, Uemura H, Yamaba S, et al. Significance of liver dysfunction associated with decreased hepatic CT attenuation values in Japanese patients with severe COVID-19. J Gastroenterol. 2020;55:1098-106.

98. Targher G, Mantovani A, Byrne CD, et al. Risk of severe illness from COVID-19 in patients with metabolic dysfunction-associated fatty liver disease and increased fibrosis scores. Gut BMJ Publ Group. 2020;69:1545-7.

99. Portincasa P, Krawczyk M, Smyk W, et al. COVID-19 and nonalcoholic fatty liver disease: two intersecting pandemics. Eur J Clin Invest. 2020;1:e13338.

100. Zayet S, Kadiane-Oussou NJ, Lepiller Q, et al. Clinical features of COVID-19 and influenza: a comparative study on Nord Franche-Comte cluster. Microbes Infect. 2020;22:481-8.

101. Leung GM, Rainer TH, Lau F-L, et al. A Clinical prediction rule for diagnosing severe acute respiratory syndrome in the emergency department. Ann Intern Med. 2004;141:333-42 ((American College of Physicians)).

102. Raj VS, Mou H, Smits SL, et al. Dipeptidyl peptidase 4 is a functional receptor for the emerging human coronavirus-EMC. Nature. 2013;495:251-4 ((Nature Publishing Group)).

103. Assiri A, Al-Tawfiq JA, Al-Rabeeah AA, et al. Epidemiological, demographic, and clinical characteristics of 47 cases of Middle East respiratory syndrome coronavirus disease from Saudi Arabia: a descriptive study. Lancet Infect Dis. 2013;13:752-61.

104. Cunney RJ, Costigan P, McNamara EB, et al. Investigation of an outbreak of gastroenteritis caused by Norwalk-like virus, using solid phase immune electron microscopy. J Hosp Infect. 2000;44:113-8.

105. Kobayashi S, Morishita T, Yamashita T, et al. A large outbreak of gastroenteritis associated with a small round structured virus among schoolchildren and teachers in Japan. Epidemiol Infect. 1991;107:81-6.

106. Minodier L, Masse S, Capai L, et al. Clinical and virological factors associated with gastrointestinal symptoms in patients with acute respiratory infection: a two-year prospective study in general practice medicine. BMC Infect Dis. 2017;17:729.

107. Singh S, Khan A, Chowdhry M, et al. Risk of severe coronavirus disease 2019 in patients with inflammatory bowel disease in the United States: a multicenter research network Study. Gastroenterology. 2019;1:S0016508520347557.

108. COVID-19: Data Main-NYC Health [Internet]. [cited 2020 Dec 7]. Available from; https://www1.nyc.gov/site/doh/covid/ covid-19-data.page

109. Raharja A, Tamara A, Kok LT. Association between ethnicity and severe COVID-19 disease: a systematic review and metaanalysis. J Racial Ethn Health Dispar. 2020;1:1-10.

110. Sekine S, Hayashi Y, Ando T, et al. Outbreaks of acute gastroenteritis caused by small round structured viruses in Tokyo. Kansenshogaku Zasshi. 1992;66:974-82.

Publisher's Note Springer Nature remains neutral with regard to jurisdictional claims in published maps and institutional affiliations. 